\title{
First record of Hyphobasidiofera malaysiana (Basidiomycota) from Vietnam
}

\section{Vadim A. Mel'nik ${ }^{1 *}$, Alina V. Alexandrova ${ }^{2,3}$, Ivan V. Zmitrovich ${ }^{1}$, Uwe Braun ${ }^{4} \&$ Eugene S. Popov ${ }^{1,3}$}

${ }^{1}$ Laboratory of the Systematics and Geography of Fungi, Komarov Botanical Institute, Russian Academy of Sciences, Professor Popov Street 2, St. Petersburg 197376, Russia

${ }^{2}$ Moscow State Lomonosov University, Department of Biology, Lenin Hill Street 1, 12, Moscow 119234, Russia ${ }^{3}$ Joint Vietnam-Russian Tropical Research and Technological Centre, Nguyen Van Huyen, Nghia Do, Cau Giau, Hanoi, Vietnam

${ }^{4}$ Martin Luther University, Institute of Biology, Department of Geobotany and Botanical Garden, Herbarium, Neuwerk 21, 06099 Halle (Saale), Germany

Received 14 April 2015 / Accepted 23 April 2015 / Published 3 May 2015

Mel'nik, V.A., Alexandrova, A.V., Zmitrovich, I.V., Braun, U. \& Popov, E.S. 2015. First record of Hyphobasidiofera malaysiana (Basidiomycota) from Vietnam. - Mycobiota 5: 1-5. doi: 10.12664/ mycobiota.2015.05.01

Abstract. The basidiomycete Hyphobasidiofera malaysiana, described from Malaysia, has been found in Vietnam for the first time. This hitherto rarely encountered species is described, illustrated and discussed.

Key words: basidiomycetes, distribution, south-east Asia, taxonomy, Vietnam

\section{Introduction}

In May 2014, A. V. Alexandrova collected leaf, fruit, bark and twig litter in the Chu Yang Sin National Park, Krong Bong District, Dak Lak Province, Vietnam. The field examinations were carried out within the framework of a Research Program of the Vietnam-Russian Tropical Research and Technology Centre. Dried samples were forwarded to V. A. Mel'nik for further treatment and identification of asexual fungi developed on the plant debris, including bark of Pinus dalatensis which seemed to be free of any fructification. In October 2014, Yu. K. Novozhilov deposited some bark in a moist chamber, in which after about two months abundant snow-white coremioid fructification was observed on the surface of the bark fragments. At first glance, the fructification was superficially reminiscent of the hyphomycete

*Corresponding author: e-mail: vadim.melnik@mail.ru 
genus Phacellium Bonord., but a closer inspection and microscopic examination readily revealed a morphologically unusual basidiomycete with synnematous structures arising from an inconspicuous subiculum and terminally giving rise to basidial clusters.

\section{Material and methods}

Fresh samples of bark of Pinus dalatensis collected in the course of field examinations in Vietnam were dried at room temperature and later deposited in a moist chamber for two months. Basidiomata developed under moist chamber conditions were later examined in distilled water and photographed using a Zeiss microscope, Stemi 2000CS, and Axio Imager A1 equipped with Nomarski differential interference contrast optics. The sizes of basidia, basidiospores and other structures are based on 30 measurements per structure with the extremes given in brackets. The examined specimens are deposited in the herbarium of the Komarov Botanical Institute, St. Petersburg, Russia (LE), and duplicates in DAOM and HAL.

\section{Results and discussion}

Hyphobasidiofera malaysiana K. Matsush. \& Matsush., Matsushima Mycological Memoirs 9: 33, 1996.

Basidiomata formed as multiple scattered synnematous knobs appearing arising from barely visible arachnoid subiculum (Figs 1, 2). Individual synnemata $0.1-0.4 \mathrm{~mm}$ diam., poorly divided into a loose head and shrinking stalk, to $0.5 \mathrm{~mm}$ long; heads composed of free basidial clusters, snow- to ivory-white. Subicular mat expanding over bark, arachnoid, snow- to ivory-white, very loose, forming thin layers (to $0.05 \mathrm{~mm}$ thick), invisible after substrate exsiccation. Hyphal system monomitic, hyphae 1.5-3(-4) $\mu \mathrm{m}$ diam., hyaline, regularly branched at an acute angle, bearing closed clamp connections (occasionally simple septa observed in zones of advanced stages); initially thin-walled, later with slightly thickened wall, smooth or sometimes finely rugose; in the subiculum rather loosely arranged, threads more or less parallel, with irregularly swollen cystidium-like appendages and ampulliform swellings; in the synnemata densely packed and furnished with basidial clusters. Basidia of apobasidium-type (with a passive spore discharge), arising as urniform to clavate probasidia, $7.5-20 \times 2.5-5 \mu \mathrm{m}$, later elongating to $30-45 \mu \mathrm{m}$ (several stages of metabasidium expansions are discernable in Fig. 3), cylindrical or tapering, apically giving rise to (2-) 4 sterigmatic pedicels (Fig. 4), with refractive content, initially thin-walled, later with slightly thickened walls, after protoplasm collapse having a cystidial appearance. Basidiospores obovate to ellipsoid, 10-16 × 7-10 $\mu \mathrm{m}$, with more or less symmetrical small (to almost inconspicuous) denticle-like hilum, with slightly thickened wall, initially hyaline, later with refractive content, smooth (Fig. 5).

Specimen examined - On bark of living tree of Pinus dalatensis (developed in a moist chamber), VIETNAM, DAK LAK PROVINCE, Krong Bong District, Chu Yang Sin National Park, $8 \mathrm{~km}$ NW of Chu Yang Sin mountain, upper montane primary evergreen tropical mixed forest, 12²4'31" N, 108 23'19" E, 21 May 2014, leg. A.V. Alexandrova (isol. Yu.K. Novozhilov), det. V.A. Mel'nik (LE 264563, HAL 2682 F, DAOM 574872). 


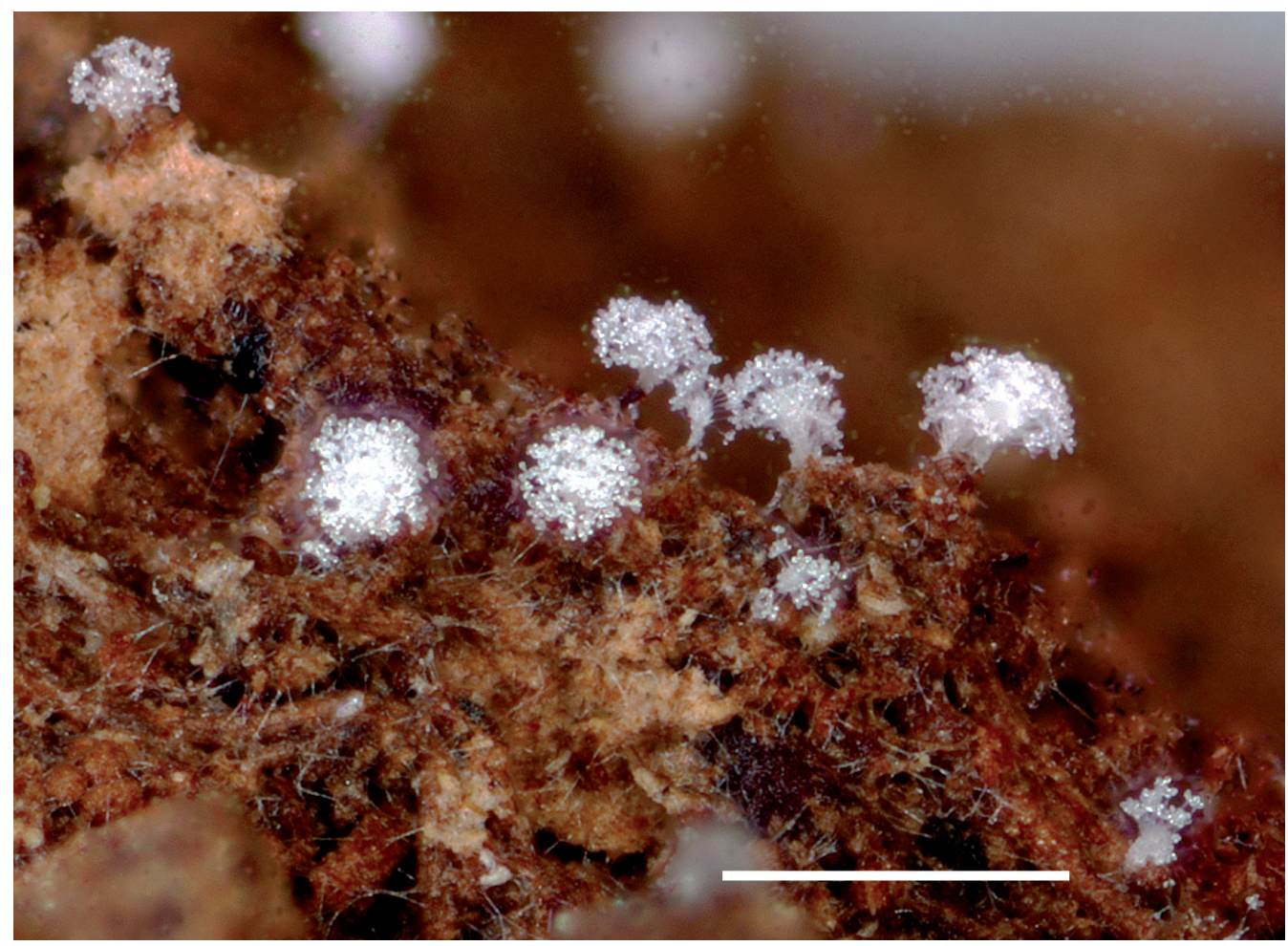

Fig. 1. Colonies of Hyphobasidiofera malaysiana on bark of Pinus dalatensis. Scale bar $=500 \mu \mathrm{m}$

Hyphobasidiofera malaysiana was described from Kuala Lumpur, Malaysia, on dead bark of an unidentified broad-leafed tree (Matsushima \& Matsushima 1996). The collection on bark of Pinus dalatensis from Vietnam agrees morphologically well with the material described from Malaysia and represents the second record of this species which supplements the knowledge on the distribution of this fungus and the mycobiota of south-east Asia in general. Attempts to cultivate the Vietnamese specimen seem to be successful, hyphae with clamps have been developed, but the fungus concerned grows very slowly.

The species is very characteristic due to its small, scattered, white basidiomata distributed over a barely visible subiculum. The development of basidiomata depends on substrate moisture and stage of development. Basidiome initials are minute (about $0.1 \mathrm{~mm}$ diam.) but nevertheless easily discernable. The heads formed in more advanced stages are initially dense, later loose, splaying out, giving the impression of a snow-white pruina. The basidiome type is neither cyphelloid nor gasteroid, but using the hyphomycete terminology it can be interpreted as synnematous structure. The subiculum is barely visible, in some areas it can be observed at $\times 4-10$ magnification only. Hyphae are typical for clamp-bearing basidiomycetes, although septation without clamps could also be observed. All hyphal elements are rather sensitive to desiccation and often collapse in the course of drying and imitate pseudoskeletal hyphae and pseudocystidia. Basidia are rather abundant at the apex of synnema-like structures and consist of several generations of probasidia which tend to 


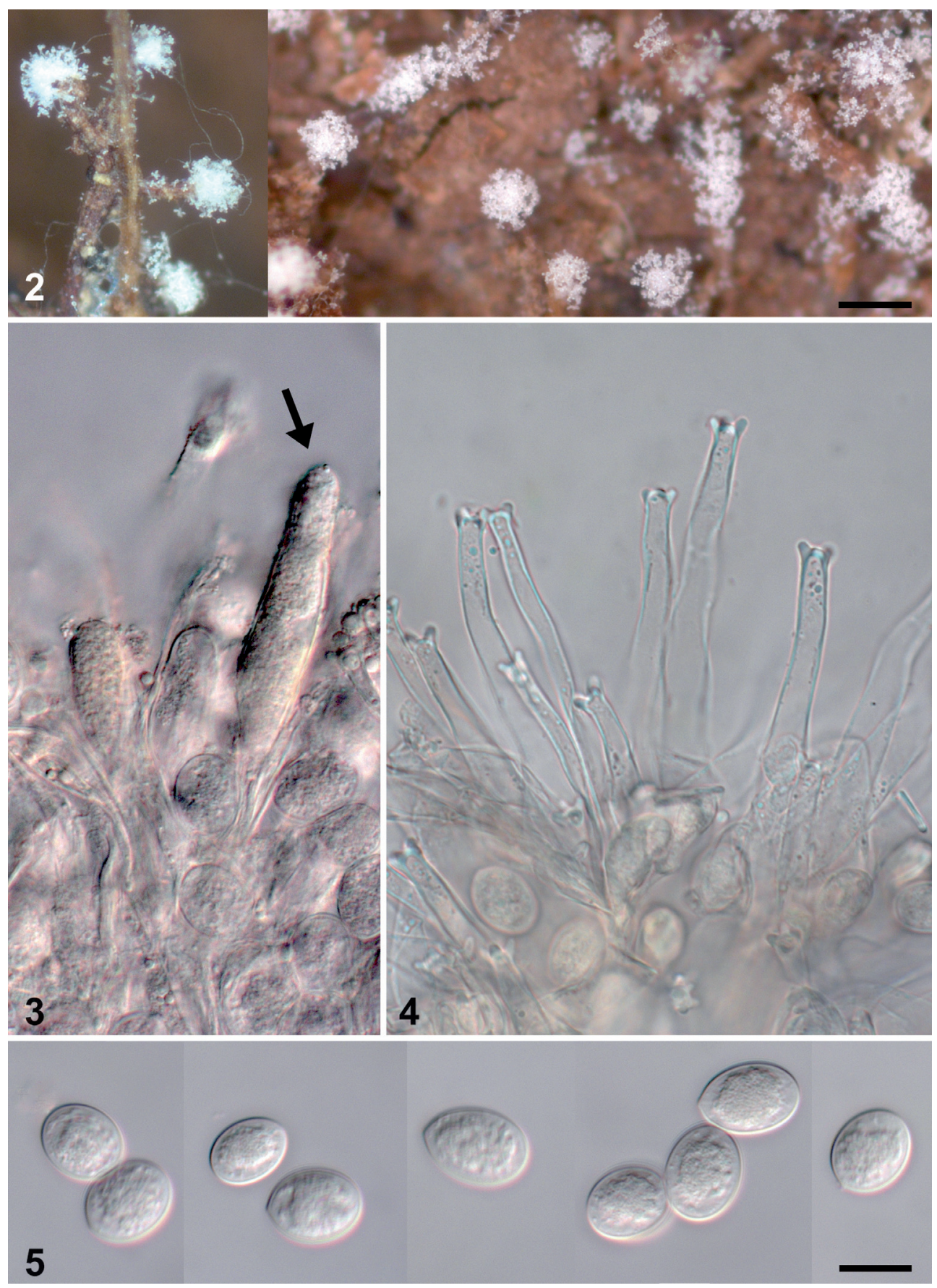

Figs 2-5. Hyphobasidiofera malaysiana (LE 264563). 2. Colonies on bark of Pinus dalatensis in LM. 3. Probasidia and elongating metabasidium (arrowed). 4. Mature basidia with sterigmata. 5. Basidiospores. Scale bars: $2=200 \mu \mathrm{m}, 3-5=10 \mu \mathrm{m}$ 
give rise to stout tapering metabasidia. Sterigmatic pedicels (usually 4) are pulled together and have a papillary appearance. The obovate or ellipsoid basidiospores with weak tendency to become lemon-shape are symmetric due to passive spore discharge.

The taxonomic status and generic affinity of Hyphobasidiofera are not yet clear. Seifert et al. (2011) cited the latter genus as synonym of Rogersiomyces J.L. Crane \& Schokn., but it was not intended to discuss and solve these problems in the present paper. This will be done elsewhere pending on cultures and results of molecular sequence analyses.

Acknowledgements. A. V. Alexandrova's field examinations were partly supported by the Russian Science Foundation (project N14-50-00029). We are much obliged to Yu. K. Novozhilov (Laboratory of the Systematics and Geography of Fungi, Komarov Botanical Institute, Russian Academy of Sciences, St. Petersburg, Russia) for getting isolates of $H$. malaysiana after treatment of the substrate in a moist chamber and to K. Matsushima (Shionogi Pharmaceutical Research Center, Japan) for confirming the identity of the species. Since thanks are due to K. Seifert (Eastern Cereal and Oilseed Research Centre, Agriculture Canada, Research Branch, Ottawa, Canada), for helpful comments and discussions.

\section{References}

Matsushima, K. \& Matsushima, T. 1996. Fragmenta Mycologica - II. - Matsushima Mycological Memoirs 9: 33-40.

Seifert, K.A., Morgan-Jones, G., Gams, W. \& Kendrick, B. 2011. The Genera of Hyphomycetes. - CBS Biodiversity Series 9: 1-997. 